\title{
Therapeutic treatment with a novel hypoxia-inducible factor hydroxylase inhibitor (TRCI60334) ameliorates murine colitis
}

This article was published in the following Dove Press journal:

Clinical and Experimental Gastroenterology

24 January 2014

Number of times this article has been viewed

\author{
Ram Gupta' \\ Anita R Chaudhary ${ }^{2}$ \\ Binita N Shah' \\ Avinash $V$ Jadhav $^{3}$ \\ Shitalkumar P Zambad' \\ Ramesh Chandra Gupta ${ }^{4}$ \\ Shailesh Deshpande ${ }^{4}$ \\ Vijay Chauthaiwale ${ }^{4}$ \\ Chaitanya Dutt ${ }^{4}$ \\ 'Department of Pharmacology, \\ ${ }^{2}$ Cellular and Molecular Biology, \\ ${ }^{3}$ Preclinical Safety Evaluation, \\ ${ }^{4}$ Discovery, Torrent Research \\ Centre, Torrent Pharmaceuticals Ltd, \\ Gandhinagar, Gujarat, India
}

Background and aim: Mucosal healing in inflammatory bowel disease (IBD) can be achieved by improvement of intestinal barrier protection. Activation of hypoxia-inducible factor (HIF) has been identified as a critical factor for barrier protection during mucosal insult and is linked with improvement in symptoms of colitis. Although prophylactic efficacy of HIF hydroxylase inhibitors in murine colitis have been established, its therapeutic efficacy in clinically relevant therapeutic settings have not been established. In the present study we aim to establish therapeutic efficacy of TRC160334, a novel HIF hydroxylase inhibitor, in animal models of colitis.

Methods: The efficacy of TRC160334 was evaluated in two different mouse models of colitis by oral route. A prophylactic efficacy study was performed in a 2,4,6-trinitrobenzene sulfonic acidinduced mouse model of colitis representing human Crohn's disease pathology. Additionally, a therapeutic efficacy study was performed in a dextran sulfate sodium-induced mouse model of colitis, a model simulating human ulcerative colitis.

Results: TRC160334 treatment resulted in significant improvement in disease end points in both models of colitis. TRC160334 treatment resulted into cytoprotective heatshock protein 70 induction in inflamed colon. TRC160334 successfully attenuated the rate of fall in body weight, disease activity index, and macroscopic and microscopic scores of colonic damage leading to overall improvement in study outcome.

Conclusion: Our findings are the first to demonstrate that therapeutic intervention with a HIF hydroxylase inhibitor ameliorates IBD in disease models. These findings highlight the potential of TRC160334 for its clinical application in the treatment of IBD.

Keywords: IBD, hypoxia-inducible factor, HIF activation

\section{Introduction}

Inflammatory bowel disease (IBD) is comprised of Crohn's disease (CD) and ulcerative colitis (UC) and is a chronic gastrointestinal inflammation disorder with considerable morbidity and health care burden. ${ }^{1}$ Although the pathophysiological understanding of IBD has enhanced over time, its exact etiology is yet to be established and the disease remains incurable. IBD remains a complex multifactorial disease involving dysfunctional intestinal epithelial barrier, aberrant innate immune system responses, contribution by intestinal flora along with genetic predisposition ${ }^{2}$ and stress. ${ }^{3}$ However, recent research indicates that breach in intestinal barrier function is one of the early and crucial factors in the pathogenesis of IBD. ${ }^{4}$ Breach of intestinal integrity allows nonselective influx of luminal antigens which acts as a trigger for immune cells in the lamina propria, resulting in prolonged, chronic inflammation ultimately leading to clinical presentation of disease in the form of abdominal pain, intestinal bleeding, weight loss, fever, and diarrhea of different severity. ${ }^{5}$
Correspondence: Vijay Chauthaiwale Torrent Research Centre, Torrent Pharmaceuticals Ltd, PO Bhat, Dist Gandhinagar, 382428 Gujarat, India Tel +917923969100 Fax +91 7923969135 Email vijaychauthaiwale@torrentpharma. com 
Current therapeutic strategies for IBD involve multidisciplinary management targeting inflammation and immune components associated with the disease. However, several limitations exist with current therapeutic options such as inadequate response or nonresponsiveness, loss of response, and immunosupression. ${ }^{5}$ More recently, approval of anti-tumor necrosis factor (TNF) $\alpha$ antibodies for IBD have revolutionized the treatment approach. However, in addition to high cost, adverse effects such as immunogenicity, infusion site reaction, and the loss of efficacy have been observed for anti-TNF $\alpha$ antibodies. ${ }^{6}$ Recently, mucosal healing has emerged as a key treatment goal in IBD and as a predictor for sustained clinical remission and-resection free survival of patients. While current therapeutic strategies for IBD reduce disease activity and improve disease symptoms, these agents are inadequate in achieving mucosal healing. ${ }^{7}$ Indeed, despite therapeutic developments, the number of IBD patients ultimately requiring surgical intervention remain high. ${ }^{8}$ This highlights the significant unmet need which can be addressed by new therapeutics aimed at effective mucosal healing for IBD. Effective blockade of breach in intestinal barrier function is expected to bring about such mucosal healing in IBD.

Under physiological conditions, intestinal tissue presents a unique steep oxygen gradient with an oxygen rich vascular bed and almost anoxic luminal epithelial interface. ${ }^{9}$ However, when inflamed, a high degree of intestinal mucosal hypoxia is observed in the animal model for colitis, an observation that has been further confirmed in human IBD patients. ${ }^{10}$ Hypoxia-inducible factor (HIF) activation is one of the prominent adaptive mechanisms associated with hypoxia/ ischemia. HIF activation induces transcription of genes in a concerted manner that performs multiple functions to adapt and to recover from hypoxic/ischemic conditions. ${ }^{11}$ Recently, we demonstrated that TRC160334, a novel HIF hydroxylase inhibitor, ameliorates ischemic acute kidney injury in rats. ${ }^{11}$ The potential of inhibiting HIF hydroxylase to bring about subsequent HIF activation has been identified for the treatment of diseases like anemia ${ }^{12}$ and IBD. ${ }^{13}$

In the context of IBD, HIF activation results in upregulation of a set of target genes linked with maintenance of intestinal barrier function (heatshock protein 70 [HSP70], multidrug resistant gene 1 , intestinal trefoil factor). ${ }^{14}$ Karhausen et $\mathrm{al}^{14}$ have shown that transgenic mice with targeted mutation for HIF-1 $\alpha$ in intestinal epithelial cells (inability to form HIF-1) demonstrate a more severe colitic phenotype. In addition, animals mutant for von Hippel-Lindau gene (forming constitutively active HIF-1) were protected from developing colitis. Pharmacological inhibition of HIF hydroxylase and subsequent HIF activation has resulted in protection of colitis in mice. ${ }^{13}$ However, this study employed only the prophylactic treatment of HIF hydroxylase inhibition and did not explore the therapeutic treatment regimen. ${ }^{13}$ In the present study, we have studied the potential of TRC160334 for treatment of colitis in clinically relevant animal models simulating human $\mathrm{CD}$ and $\mathrm{UC}$ disease conditions using prophylactic as well as therapeutic treatment regimens.

\section{Methods TRCI 60334}

TRC160334 is a novel HIF hydroxylases inhibitor and its chemical name is [(2-hydroxy-4-oxo-6,7,8,9-tetrahydro4H,5H-10-thia-1,4a-diaza-benzo[a]azulene-3-carbonyl)amino]-acetic acid. The chemistry in terms of design, synthesis, and biological activity of HIF activation by TRC160334 have been described previously. ${ }^{11}$

\section{In vivo studies}

\section{Animals}

In-house bred BALB/c mice ( 7 to 11 week of age) were used for the study. Mice were housed in individually ventilated cages, maintained on 12-hour light-dark cycles, and had access to Harlan-2014c rodent chow (Harlan Laboratories, Inc., Madison, WI, USA) and water ad libitum. The individually ventilated cages were maintained under a controlled environment of temperature $22^{\circ} \mathrm{C} \pm 3^{\circ} \mathrm{C}$, relative humidity $30 \%-70 \%$, and air exchange rate of $40-50$ air changes per hour. Animal care and all experimental procedures were carried out in accordance with guidelines of the Committee for the Purpose of Control and Supervision of Experiments on Animals, Government of India. ${ }^{15}$ The study protocols were approved by the Institutional Animal Ethics Committee of Torrent Pharmaceuticals Limited at Gujarat, India.

\section{2,4,6-trinitrobenzene sulfonic acid (TNBS)-induced murine colitis model}

Male BALB/c mice, based on their body weights, were randomized into two groups: group 1 consisted of TNBS colitis mice receiving vehicle $(\mathrm{n}=22)$ and group 2 consisted of TNBS colitis mice treated with TRC160334 $(\mathrm{n}=12)$. On day 0 under isofluorane anesthesia (Forane; Aesica Queenborough Ltd., Kent, UK), acute colitis was induced in 16 hour fasted mice by an intrarectal administration of $1.5 \mathrm{mg}$ TNBS (Sigma-

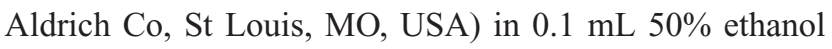
through a flexible catheter $4 \mathrm{~cm}$ proximal to the anus. To ensure proper distribution of TNBS within the colon and to prevent reflex leakage, mice were kept in a vertical head 
down position for 45 seconds after instillation. Six control mice underwent identical procedures but were instilled with $50 \%$ ethanol.

\section{Prophylactic efficacy of TRCI 60334 in TNBS-induced murine colitis}

Treatment with TRC160334, $2 \mathrm{mg} / \mathrm{kg} /$ day, per oral (po) was initiated a day prior to induction of colitis (day -1) and continued until study termination, ie, day 4 (Figure 1A). Deaths occurring within 24 hours of TNBS instillation were not included in study. ${ }^{14}$

\section{Dextran sulfate sodium (DSS)-induced murine colitis model}

Autoclaved drinking water with $5 \%$ dissolved DSS (molecular weight 36,000-50,000 Da; MP Biomedicals LLC, Solon, OH, USA) was provided ad libitum to female BALB/c mice for induction of colitis. Fresh DSS solution was provided every alternate day. DSS was provided to mice until study day 11, followed by three DSS free days, ie, until day 14, the study termination day. On day 5 , mice were randomized into three groups based on their body weight and severity of disease activity index (DAI): group 1 consisted of DSS colitis mice receiving vehicle ( $\mathrm{n}=9)$, and groups 2 and 3 were DSS colitis mice treated with TRC160334 at $2 \mathrm{mg} / \mathrm{kg} /$ day $(\mathrm{n}=9)$ or $5 \mathrm{mg} / \mathrm{kg} /$ day ( $\mathrm{n}=9$ ), respectively. Five control mice underwent identical procedures and received autoclaved drinking water throughout the study duration.

\section{Therapeutic efficacy of TRCI 60334 in DSS-induced murine colitis}

Treatment with TRC160334 at 2 and $5 \mathrm{mg} / \mathrm{kg} /$ day, po was initiated from day 5 and continued until study termination (Figure 1B). Only mice that demonstrated an initial response to DSS treatment were included in the study; this response was defined as a fall in mean body weight (day 1 to day 5 ) by $\geq 1 \%$.

\section{Assessment of inflammation and colitis severity}

DAI for colitis

For assessment of clinical severity of colitis, DAI was calculated as reported earlier. ${ }^{16,17}$ DAI is a composite

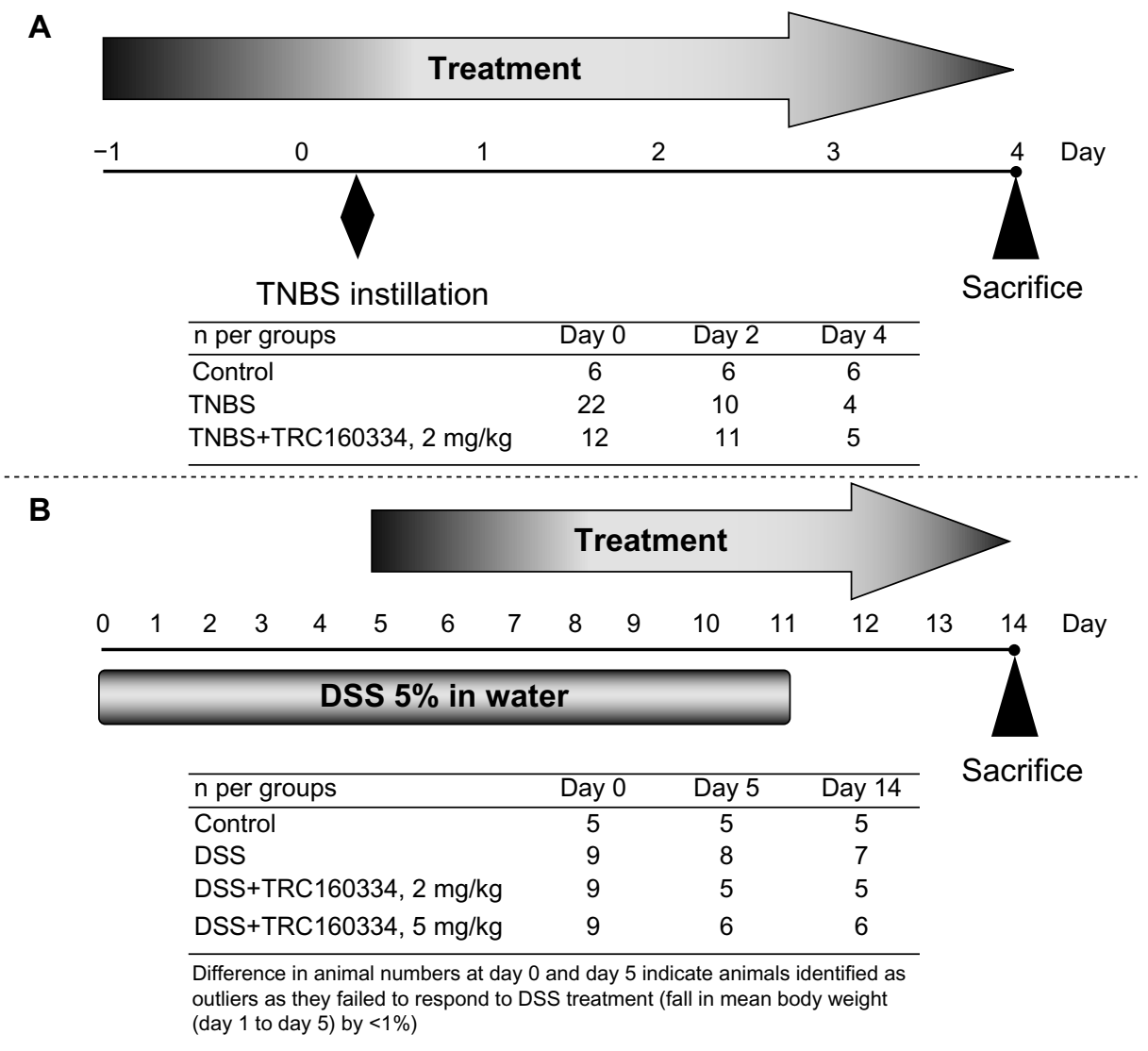

Figure I Scheme of the experimental protocol for efficacy evaluation of TRCI60334 in a mouse model of colitis.

Notes: (A) 2,4,6-trinitrobenzene sulfonic-induced colitis and (B) dextran sulfate sodium-induced colitis.

Abbreviations: DSS, dextran sulfate sodium; TNBS, 2,4,6-trinitrobenzene sulfonic acid. 
score derived on the basis of loss in body weight, stool consistency, and appearance of blood in feces. Briefly, body weights were monitored daily, whereas stool consistency and rectal bleeding were examined every alternate day. The loss in body weight was scored as: 0 , no weight loss; 1 , weight loss of $1 \%$ to $5 \%$; 2 , weight loss of $6 \%$ to $10 \%$; 3 , weight loss of $11 \%$ to $20 \%$; and 4 , weight loss $>20 \%$. The assessment of diarrhea (stool consistency) was scored as 0 for normally formed pellets; 2 for pasty and semiformed pellets; and 4 for liquid stools. Bleeding was scored using the standard Guaiac paper test (Hemospot; Crest Biosystems, Goa, India) as follows: 0, negative hemoccult; 2, positive hemoccult; and 4, visible blood/ gross bleeding from the rectum. These scoring parameters were added together resulting in a total clinical score ranging from 0 (healthy) to 12 (maximal ill/activity of colitis). At the end of the experiment, the colons were harvested for further macroscopic/microscopic damage scoring and expression study.

\section{Macroscopic assessment of colonic damage}

The assessment of the macroscopic colonic damage was performed using a slightly modified Morris score. ${ }^{18}$ The colon was excised, opened longitudinally, and washed in cold phosphate buffered saline. Features of macroscopic damage were graded as follows: 0 , no ulcer and no inflammation; 1 , focal hyperemia, but no ulcer; 2, severe hyperemia; 3, ulceration and inflammation at one site only; 4 , ulceration and inflammation at two or more sites; 5 , ulceration extending $1 \mathrm{~cm}$ but less than $2 \mathrm{~cm}$; 6 to 10 , score increased by 1 point for increase of every $1 \mathrm{~cm}$ in lesion area up to maximum score of 10 .

\section{Histological evaluation of colonic damage}

The colonic tissues were fixed in 10\% neutral buffered formalin, processed, sectioned at $4 \mu \mathrm{m}$ thickness, and stained with a routine hematoxylin and eosin method. ${ }^{19}$ Stained sections were evaluated in a blinded manner by a pathologist.

Sections from TNBS treated mice were semiquantified for severity of lesion using a score of 0 to 6: 0 , normal crypt architecture; 1 , focal to multifocal inflammatory cell infiltration or focal necrosis; 2, diffuse inflammatory cell infiltration or focal to multifocal erosions with necrosis extending up to muscularis mucosa; 3 , multifocal erosions or ulceration with and without partial loss of crypts along with inflammatory cell infiltration; 4, diffuse erosions or focal to multifocal ulceration along with partial loss of crypts and inflammatory cell infiltration; 5, multifocal to diffuse ulceration with necrosis extending up to serosa, complete loss of crypts, inflammatory cell infiltration, and mineralization in mucosa and submucosa; 6, multifocal to diffuse ulceration with necrosis perforating serosa, complete loss of crypts, inflammatory cell infiltration, and mineralization in mucosa and submucosa.

Sections from DSS treated mice were semiquantified for severity of lesion using a scoring of 0 to 4 : 0 , normal crypt architecture; 1, crypt shortening, loss of basal one third crypt, lamina propria prominent with minimal inflammatory changes; 2, crypt shortening, loss of basal two third crypt, thinning of epithelium with mild inflammatory changes; 3 , loss of entire crypt with intact epithelial layer and moderate inflammatory changes; 4, loss of entire crypt along with the epithelial layer (erosion) with severe inflammatory changes..$^{20,21}$

\section{Evaluation of gene expression}

Colon tissue of vehicle and TRC160334 treated animals were processed and total RNA was isolated. Expression of interleukin (IL)-10, TNF- $\alpha$, and interferon (INF)- $\gamma$ mRNA along with expression of $18 \mathrm{~S}$ rRNA was monitored employing gene specific primer and probes (Applied Biosystems, Foster City, CA, USA) by quantitative real-time polymerase chain reaction on ABI 7900 HT (Applied Biosystems, Foster City, CA, USA). mRNA expression was normalized relative to the expression of $18 \mathrm{~S}$ rRNA. The results were expressed as fold induction relative to DSS control.

Colon tissue of vehicle and TRC160334 treated animals were processed for whole cell extract preparation. Proteins were separated on sodium dodecyl sulfate polyacrylamide electrophoresis followed by immunoblotting employing HSP70 antibody (SPA-810; Stressgen, Farmingdale, NY, USA) and $\beta$-actin antibody (SC47778; Santa Cruz Biotechnology, Inc, Santa Cruz, CA, USA).

\section{Statistical analysis}

Unless mentioned, results are expressed as mean \pm standard error of the mean. The difference between the means of two treatment groups was assessed using Student's $t$-test. Oneway analysis of variance with Dunnett's post hoc test was used for comparing more than two treatment groups. Values exceeding 95\% critical limits $(P<0.05)$ were considered to be statistically significant. Statistical analysis has been performed using statistical analysis system (SAS, version 9.1; SAS Institute Inc, Cary, NC, USA) and Prism (version 3.0; GraphPad Software Inc, La Jolla, CA, USA). 


\section{Results}

Prophylactic treatment with TRCI 60334 is protective in TNBS-induced colitis Improvement of DAI

Induction of colitis caused hemoccult positive soft stools along with sustained body weight loss, an indicator of disease severity, whereas no such signs were observed in control group animals. Treatment with TRC160334 significantly reduced DAI, measured at day 2 and day $4(9.9 \%$ and $33.6 \%$, respectively) with respect to vehicle treated animals (Figure 2A).

\section{Amelioration of colonic damage}

Treatment with TRC160334 remarkably attenuated severity and extent of colonic damage in comparison to vehicle treated animals. This improvement is also reflected by significantly less colonic damage observed with TRC160334 treatment in comparison to vehicle treated animals when assessed in the form of macroscopic damage scores and histological scores (Figure 2B and C). Histopathological analysis of vehicle treated colitis mice revealed colonic lesions ranging from

A

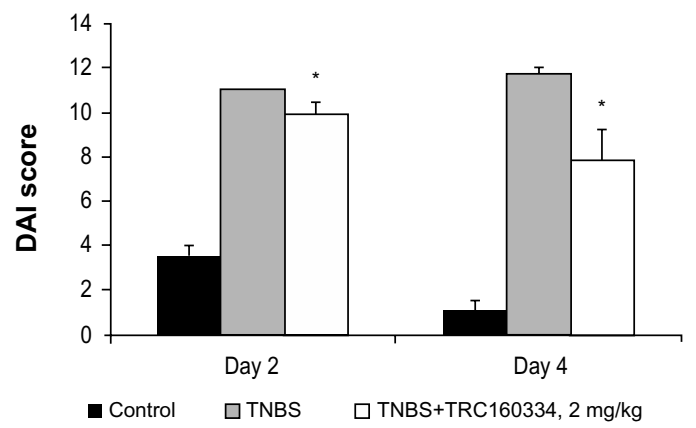

C

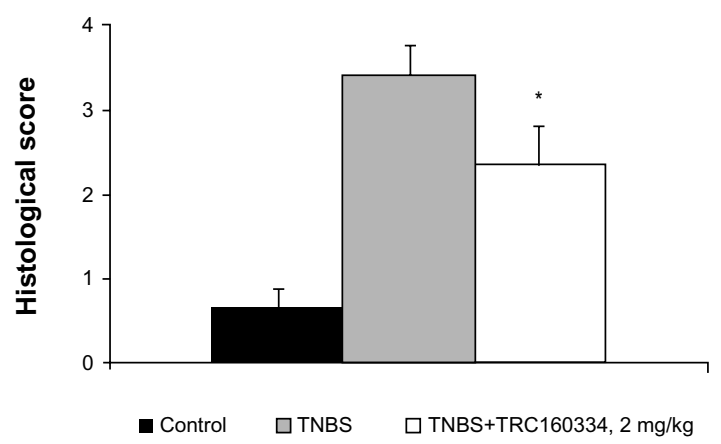

lymphocytic and/or polymorphonuclear cell infiltration, edema, partial to complete loss of crypts, and erosion to ulceration. Treatment with TRC160334 resulted in a significant reduction in severity of inflammatory and necrotic changes. A few mice in the TRC160334 treatment group also demonstrated regeneration of mucosal epithelium as the reparative response (Figure $3 \mathrm{~A}-\mathrm{C}$ ).

\section{Amelioration of body weight loss}

Rectal TNBS caused approximately $10 \%$ loss in body weight within 24 hours of instillation. Treatment with TRC160334 significantly prevented loss in body weight from day 2 until study termination in comparison to vehicle treated animals (Figure S1). Percent reduction in body weights observed on day 4 was $13.1 \% \pm 1.8 \%$ for TRC160334 treated animals as compared to $21.6 \% \pm 1.6 \%$ in vehicle treated animals (Figure 2D).

\section{Improvement in survival rate}

The combination of effect produced by TRC160334 by virtue of its ability to improve DAI and reduce colonic damage has

\section{B}

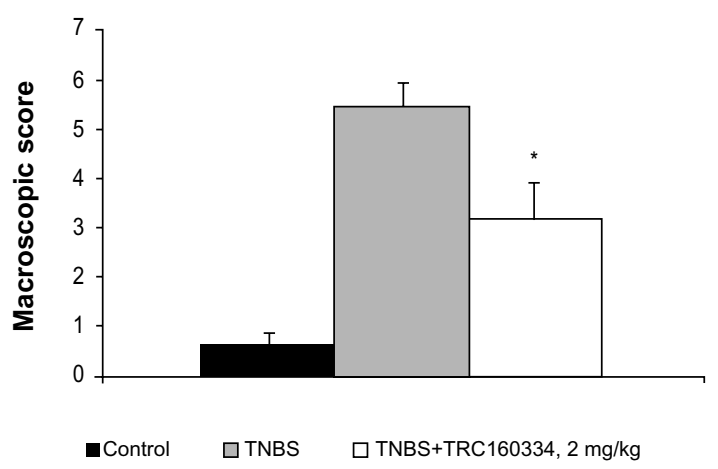

D

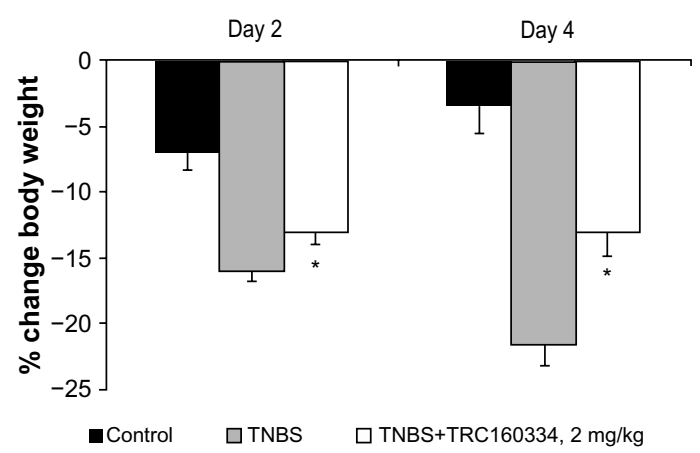

Figure 2 Treatment with TRCI60334 resulted in improvement in end points of 2,4,6-trinitrobenzene sulfonic acid-induced colitis in male BALB/c mice. Notes: (A) Mean disease activity index score; (B) mean macroscopic score; (C) mean histological score; and (D) mean percent change in body weight. Data represent mean \pm standard error of the mean. * denotes $P<0.05$ versus the 2,4,6-trinitrobenzene sulfonic acid group using Student's $t$-test. Abbreviations: TNBS, 2,4,6-trinitrobenzene sulfonic acid; DAl, disease activity index. 


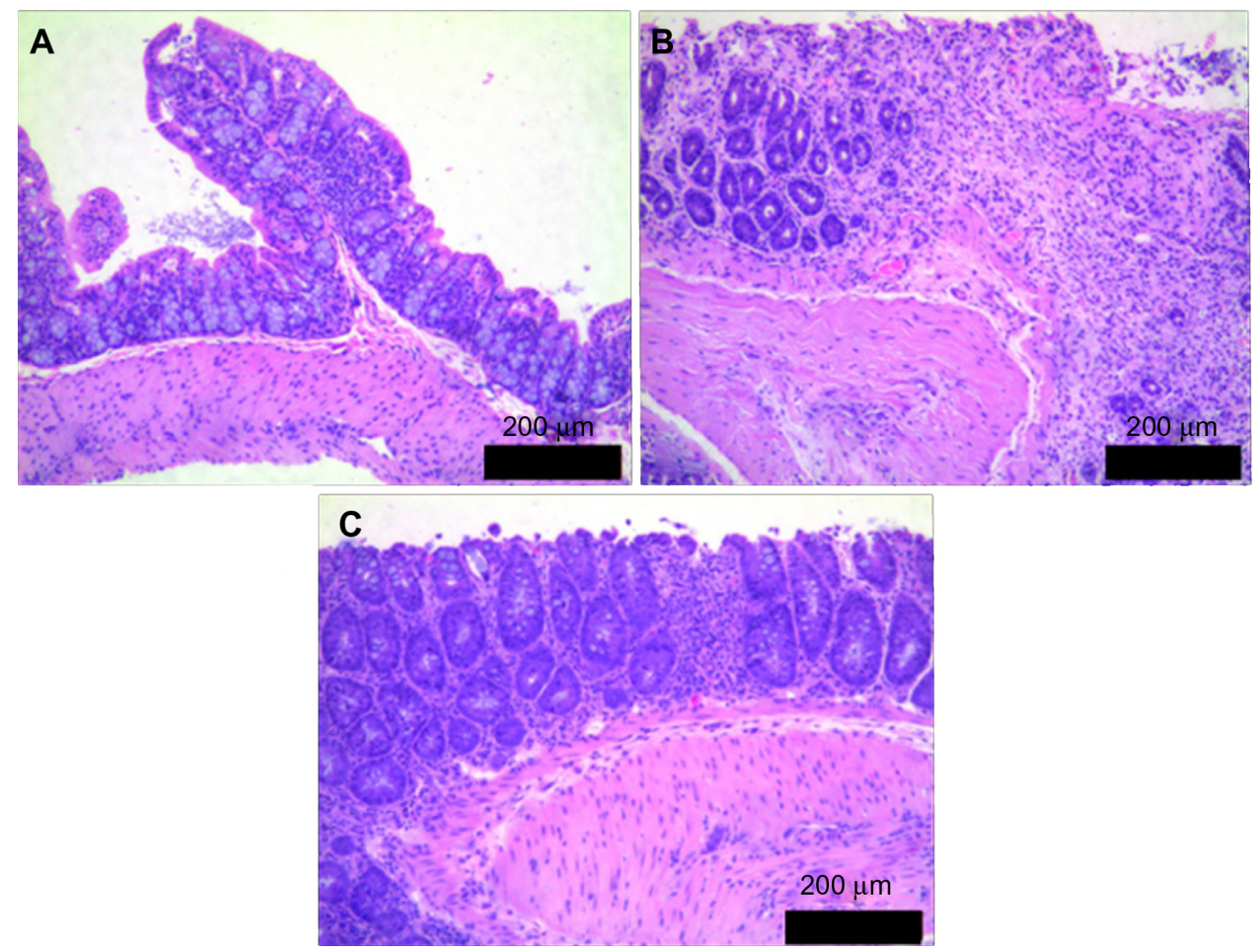

Figure 3 Hematoxylin and eosin stained representative photomicrographs for colonic histology observed in mice.

Notes: (A) Control male BALB/c mice colon with intact crypts and surface epithelium with minimal lymphocytic infiltration; (B) the colons of 2,4,6-trinitrobenzene sulfonic acid-treated mice showing partial loss of crypts along with destruction of the epithelial architecture, necrosis, and lymphocytic infiltration; and (C) TRCl60334 at 2 mg/kg resulted in reduction of severity in inflammatory and necrotic changes along with regenerating mucosal epithelium. All photomicrographs at I0× magnification.

resulted in a significantly better survival rate. The survival rate after treatment with TRC160334 was $42 \%$ compared to $18 \%$ in vehicle treated animals.

\section{Therapeutic treatment with TRCI60334 is protective in DSS-induced colitis Improvement of DAI}

Induction of colitis with 5\% DSS in drinking water resulted in a progressive increase in DAI score from day 2, reaching a maximum by day 10 . The control group of animals showed no change in DAI score (score $=0$ ). In vehicle group animals receiving DSS, the score was maintained on day 12 followed by a slight reduction on day 14 . Treatment with TRC160334, which was initiated from day 5 , successfully prevented the increment in both the rate and extent of DAI score from as early as day 8 and this trend continued until study termination (Figures 4A and S2).

\section{Reduction in colonic damage}

All mice exposed to DSS developed colonic lesions characteristic of colitis which included loss of crypts, infiltration of inflammatory cells into the mucosa and submucosa, and erosion and ulceration of graded severity. Vehicle treated animals developed a moderate to severe degree of colitis compared to control animals $(3.71 \pm 0.18$ vs $0.0 \pm 0.0)$. Treatment with TRC160334 resulted in a dose related reduction in colitis score (Figure 4B). TRC160334 treated animals showed healthier epithelial lining when compared to vehicle treated animals. Moreover, regeneration and proliferation of basal epithelial cells as a reparative response to injury was also observed in the majority of TRC160334 treated animals (Figure 5A to D).

\section{Induction of cytoprotective HSP70}

Treatment with TRC160334 resulted in a pronounced induction of HSP70 protein in the colon when compared with the respective vehicle control (Figure 4C). Trends in reduction of mRNA expression in the proinflammatory cytokines, TNF $\alpha$ and INF $\gamma$, were observed after TRC160334 treatment. Additionally, elevation of expression in the anti-inflammatory cytokine, IL-10, was also observed after TRC160334 treatment (Figure S3).

\section{Reduction in body weight loss}

Animals started showing a trend in loss of body weight as early as day 1 after DSS treatment. This trend continued with vehicle treated animals until day 11 . Vehicle treated animals 
A

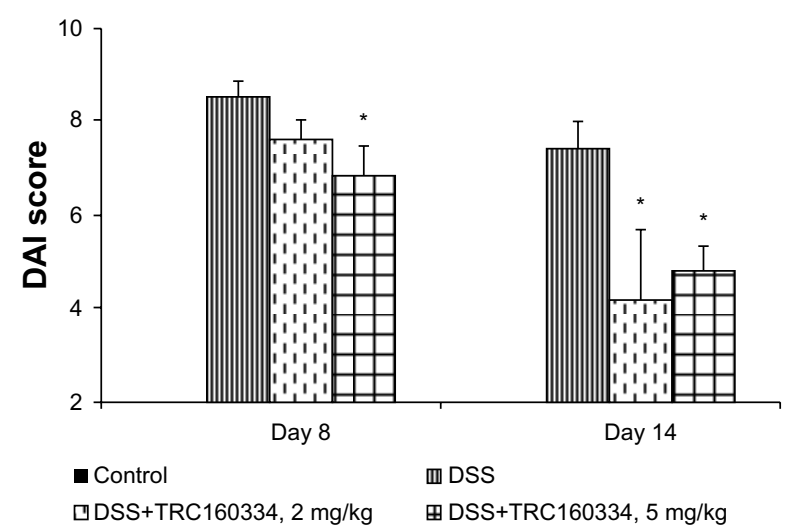

C

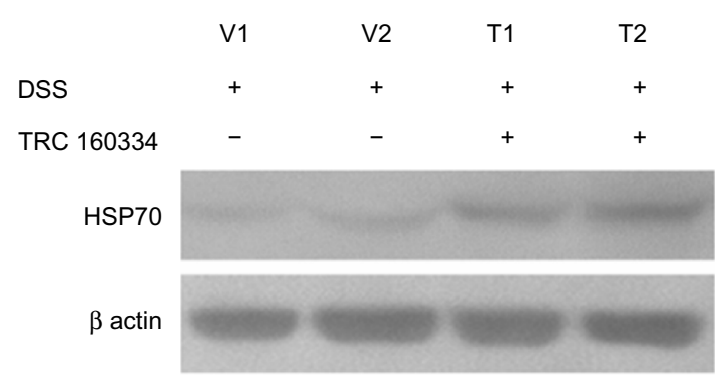

B

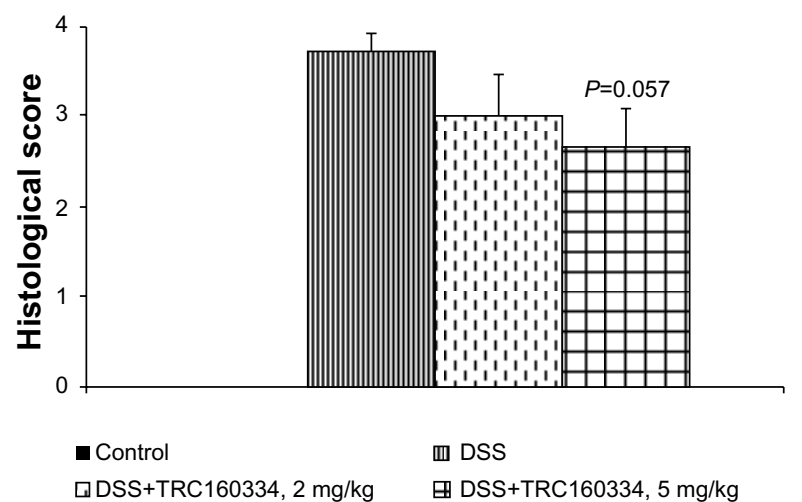

D

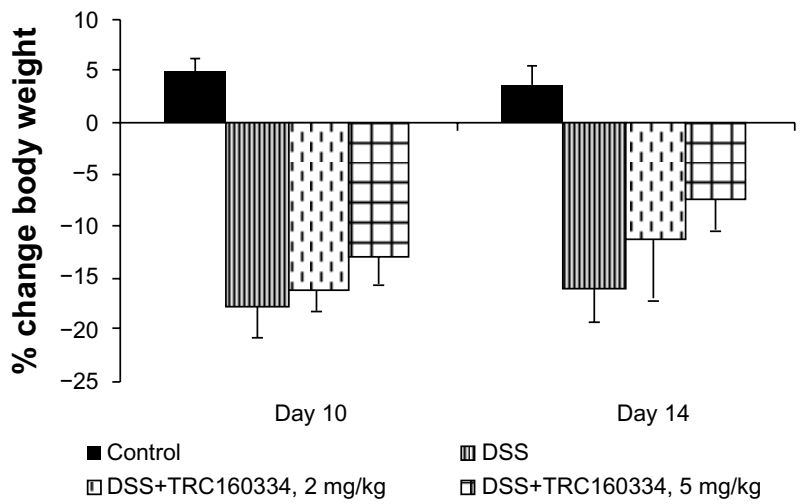

Figure 4 Treatment with TRCI60334 resulted in improvement in end points of dextran sulfate sodium-induced colitis in female BALB/c mice.

Notes: (A) Mean disease activity index score; (B) mean histological score; (C) representative immunoblot showing induction of heatshock protein 70 protein in colon, ( $\mathrm{VI}$ and V2 are dextran sulfate sodium treated animals, TI and T2 are dextran sulfate sodium + TRCI60334 treated animals); and (D) mean percent change in body weight. Data represent mean \pm standard error of the mean. * denotes $P<0.05$ versus the dextran sulfate sodium group using analysis of variance followed by Dunnett's post hoc analysis. Abbreviations: DAl, disease activity index; DSS, dextran sulfate sodium; HSP70, heatshock protein 70.

showed some gain in body weight indicating initiation of a recovery phase from day 12 onwards (after withdrawing DSS). Treatment with TRC160334 retarded the rate of loss in body weight in comparison to vehicle treated animals in a dose dependent manner. This was evident from day 6 with the TRC160334 at $5 \mathrm{mg} / \mathrm{kg} /$ day group whereas the TRC160334 $2 \mathrm{mg} / \mathrm{kg}$ /day group demonstrated this trend from day 12 (Figures 4D and S4).

\section{Discussion}

Recent advances in the pathophysiology of IBD have revealed that HIF activation may be beneficial in IBD. ${ }^{13,14}$ In the present study, we first demonstrated that prophylactic treatment with TRC160334, a novel HIF hydroxylase inhibitor, is protective in TNBS-induced colitis. Further, we demonstrated efficacy of TRC160334 in a clinically relevant therapeutic setting with the DSS-induced colitis model. The disease attenuating potential of TRC160334 in animal models of CD
(TNBS-induced colitis) ${ }^{14,22}$ and UC (DSS-induced colitis) $)^{23,24}$ at remarkably low doses via an oral route makes TRC160334 an attractive prospective therapeutic for the treatment of IBD.

TNBS-induced colitis is a clinically relevant model of $\mathrm{CD}$ as it displays similar functional and anatomical microvascular abnormalities as observed in human CD. ${ }^{14,22}$ Treatment with TRC160334 results in amelioration of body weight loss, significant improvement in DAI, reduction in colon injury severity, and better survival rate in TNBSinduced colitis. However, prophylactic treatment has limited clinical applicability as in most of the cases of IBD, treatment is initiated upon clinical disease presentation and in such cases therapeutic intervention rather than a preventive treatment strategy is warranted. The therapeutic efficacy of HIF hydroxylase inhibitors in clinically relevant therapeutic settings for colitis has not been reported. Therefore, we explored the therapeutic intervention of TRC160334 in a DSS-induced colitis model. 


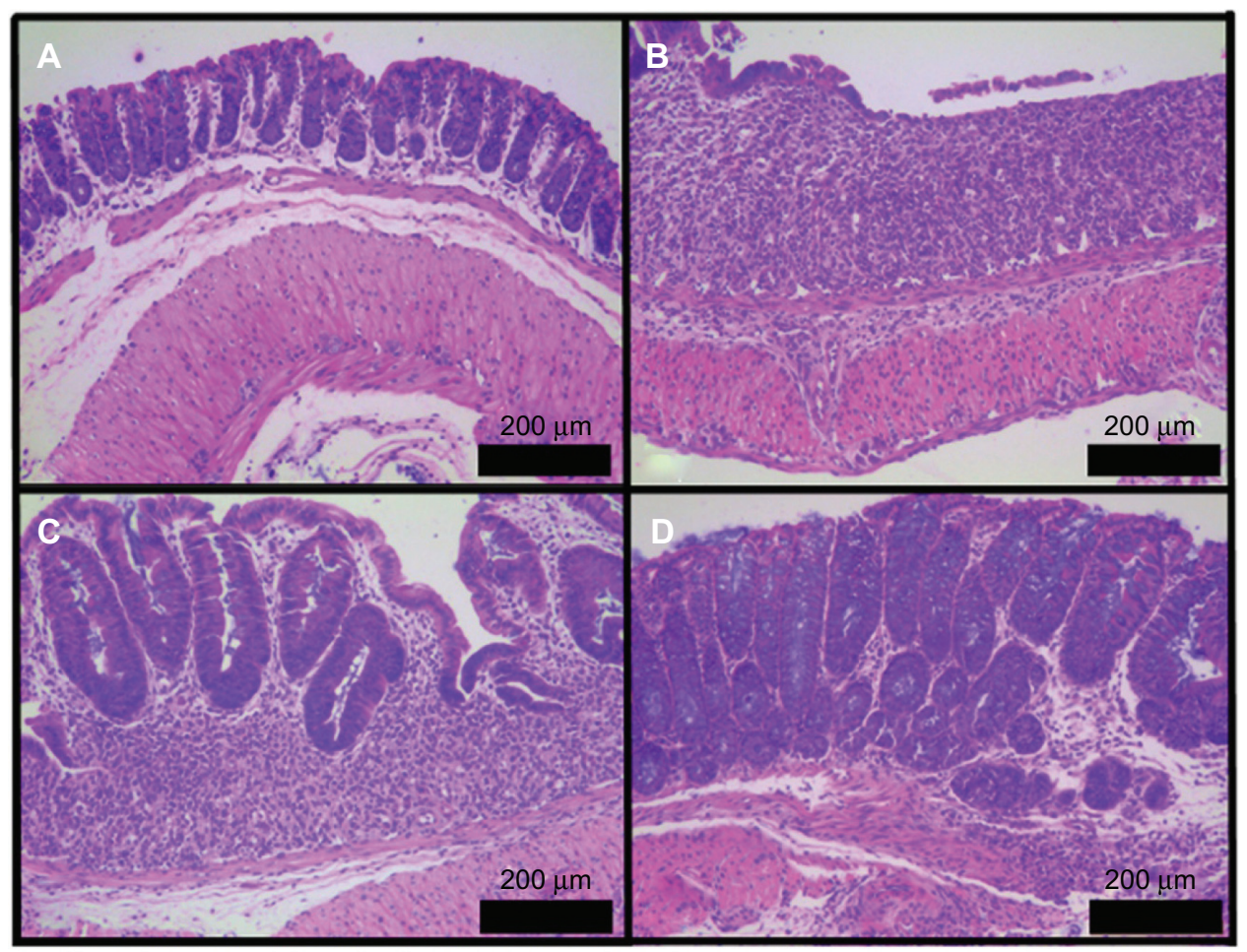

Figure 5 Hematoxylin and eosin stained representative photomicrographs of colonic histology observed in mice.

Notes: (A) Control female BALB/c mice colon with intact crypts and surface epithelium; (B) the colons of dextran sulfate sodium-treated mice showing almost complete loss of crypts along with destruction of the epithelial architecture and integrity along with intense inflammatory cellular infiltration in all layers; TRCI60334 at (C) 2 mg/kg/day and (D) $5 \mathrm{mg} / \mathrm{kg} /$ day resulted in dose dependent improvement in crypt architecture, regenerating epithelial layer along with a marked decrease in inflammatory cells. All photomicrographs at $10 \times$ magnification.

In the present study, we demonstrated that therapeutic treatment with TRC160334 is protective in DSS-induced colitis. The DSS-induced colitis model is a clinically relevant model of UC. DSS induces intestinal barrier dysfunction, stimulating local and systemic inflammation and thus reproduces many of the histopathologic and clinical features of human colitis. ${ }^{23,24}$

Treatment with TRC160334 was initiated on day 5 of DSS induction after ascertaining that DAI had reached a value of around $50 \%$ of its maximum attainable value. We continued DSS treatment for a duration of 11 days and then kept three DSS free days in our protocol. This ensured that the trigger which leads to an acute episode of colitis is still present while the treatment is initiated and continued, thus enabling us to explore the therapeutic potential of TRC160334 in the disease model. TRC160334 treatment resulted in improvement in body weight loss and DAI, along with improvement in colon histopathology in DSS-induced colitis. This finding is the first to demonstrate that therapeutic intervention with a HIF hydroxylase inhibitor is efficacious in a colitis model.

Therapeutic treatment with TRC160334 favorably impacted inflammation in the colitis model as trends of reduction in expression of the proinflammatory cytokines, TNF $\alpha$ and INF $\gamma$, and elevation in the expression of the antiinflammatory cytokine, IL-10, were observed. Treatment with TRC160334 also resulted in a significant increase in HSP70 expression which is one HIF target protein. Interventions leading to enhanced HSP70 response have been suggested as a potential approach to improve outcomes of IBD. ${ }^{25} \mathrm{HSP} 70$ transgenic mice demonstrated significantly milder colitis in response to DSS than wild type. ${ }^{26}$ HSP70 expression is found to be significantly reduced in actively inflamed mucosa from both human IBD biopsies and IL-10 $0^{-/-}$ mice with spontaneous colitis. ${ }^{27}$ Intestinal epithelial HSP70 plays an important role in protecting mucosal integrity and function by stabilizing the tight junctions between intestinal epithelial cells. Such intestinal epithelial protection is associated with restricted bacterial translocation and a reduction in inflammation. ${ }^{28}$ Conversely, compromised expression of HSP70 renders the mucosa highly susceptible to inflammatory and immune processes, and to potentially otherwise harmless enteric flora. ${ }^{27}$

An earlier report with another HIF hydroxylase inhibitor, FG-4497, has shown a beneficial effect of HIF activation by attenuating TNBS-induced colitis. ${ }^{13}$ However, benefits upon 
therapeutic treatment with FG-4497 are not known. Secondly, the prophylactic efficacy of FG-4497 was shown at higher doses (in a dose range of 20 to $40 \mathrm{mg} / \mathrm{kg} /$ day) and by an intraperitoneal route of administration, ${ }^{13}$ whereas TRC160334, as reported in current study, has shown both prophylactic and therapeutic benefits in relevant animal models of CD and UC, respectively. Additionally, comparatively lower doses and ease of administration by oral route is likely to improve compliance and convenience in clinical settings.

Our findings with TRC160334, a novel HIF hydroxylase inhibitor, confirms a prophylactic effect of such inhibitors for IBD. In addition, our findings are the first to demonstrate that therapeutic intervention with a HIF hydroxylase inhibitor can also ameliorate IBD. These findings highlight the potential of TRC160334 for its clinical application in the treatment of IBD.

\section{Acknowledgments}

The authors would like to thank Mr Prakash Dhamecha and Ms Kinjal Patel for assisting in the experimental work and Mr Ajay Shivalkar for statistical analysis.

\section{Disclosure}

The authors declare that no conflict of interest exists and they are all employees of Torrent Pharmaceuticals Ltd, India. The study is supported by Torrent Pharmaceuticals Ltd, India.

\section{References}

1. Cosnes J, Gower-Rousseau C, Seksik P, Cortot A. Epidemiology and natural history of inflammatory bowel diseases. Gastroenterology. 2011;140(6):1785-1794.

2. Abraham $\mathrm{C}$, Cho JH. Inflammatory bowel disease. $N$ Engl J Med. 2009;361(21):2066-2078.

3. Mawdsley JE, Rampton DS. Psychological stress in IBD: new insights into pathogenic and therapeutic implications. Gut. 2005;54(10): 14811-11491.

4. Hindryckx P, Laukens D, De Vos M. Boosting the hypoxia-induced adaptive response in inflammatory bowel disease: a novel concept of treatment. Inflamm Bowel Dis. 2011;17(9):2019-2022.

5. Cummins EP, Doherty GA, Taylor CT. Hydroxylases as therapeutic targets in inflammatory bowel disease. Lab Invest. 2013;93(4): 378-383.

6. Shah B, Mayer L. Current status of monoclonal antibody therapy for the treatment of inflammatory bowel disease. Expert Rev Clin Immunol. 2010;6(4):607-620.

7. Neurath MF, Travis SP. Mucosal healing in inflammatory bowel diseases: a systematic review. Gut. 2012;61(11):1619-1635.

8. Wilson BS, Lönnfors S, Vermeire S, et al. The True Impact of IBD: A European Crohn's and Ulcerative Colitis Patient Life. IMPACT Survey 2010-2011. Available from: http://efcca-solutions.net/media/ jointhefight/ImpactReport.pdf. Accessed June 4, 2013.
9. Colgan SP, Taylor CT. Hypoxia: an alarm signal during intestinal inflammation. Nat Rev Gastroenterol Hepatol. 2010;7(5):281-287.

10. Glover LE, Colgan SP. Hypoxia and metabolic factors that influence inflammatory bowel disease pathogenesis. Gastroenterology. 2011;140(6):1748-1755.

11. Jamadarkhana P, Chaudhary A, Chhipa L, et al. Treatment with a novel hypoxia-inducible factor hydroxylase inhibitor (TRC160334) ameliorates ischemic acute kidney injury. Am J Nephrol. 2012;36(3):208-218.

12. Bernhardt WM, Wiesener MS, Scigalla P, et al. Inhibition of prolyl hydroxylases increases erythropoietin production in ESRD. J Am Soc Nephrol. 2010;21(3):2151-2156.

13. Robinson A, Keely S, Karhausen J, Gerich ME, Furuta GT, Colgan SP. Mucosal protection by hypoxia-inducible factor prolyl hydroxylase inhibition. Gastroenterology. 2008;134(1):145-155.

14. Karhausen J, Furuta GT, Tomaszewski JE, Johnson RS, Colgan SP, Haase VH. Epithelial hypoxia-inducible factor-1 is protective in murine experimental colitis. J Clin Invest. 2004;114(8):1098-1106.

15. Committee for the Purpose of Control and Supervision on Experiments on Animals. CPCSEA Guidelines for Laboratory Animal Facility. Ind J Pharmacol. 2003;35(4):257-274.

16. Daniel C, Sartory NA, Zahn N, Radeke HH, Stein JM. Immune modulatory treatment of trinitrobenzene sulfonic acid colitis with calcitriol is associated with a change of a T helper (Th) $1 /$ Th17 to a Th2 and regulatory T cell profile. J Pharmacol Exp Ther. 2008;324(1): 23-33.

17. Zhang DK, Yu JJ, Li YM, et al. A Picrorhiza kurroa derivative, picroliv, attenuates the development of dextran-sulfate-sodium-induced colitis in mice. Mediators Inflamm. 2012;2012:751629.

18. Morris GP, Beck PL, Herridge MS, Depew WT, Szewczuk MR, Wallace JL. Hapten-induced model of chronic inflammation and ulceration in the rat colon. Gastroenterology. 1989;96(3):795-803.

19. Luna LG. Manual of Histologic Staining Methods of the Armed Forces Institute of Pathology. 3rd ed. New York: McGraw-Hill; 1968.

20. Kitajima S, Takuma S, Morimoto M. Histological analysis of murine colitis induced by dextran sulfate sodium of different molecular weights. Exp Anim. 2000;49(1):9-15.

21. Murano N, Murano M, Ishida K, et al. Therapeutic effect of SHI-219, a novel water soluble prodrug of EG626 (phtalazinol), on mouse dextran sodium sulfate-induced colitis. Bull Osaka Med Coll. 2006;52(2): 69-79.

22. Neurath M, Fuss I, Strober W. TNBS-colitis. Int Rev Immunol. 2000;19(1):51-62.

23. Dieleman LA, Palmen MJ, Akol H, et al. Chronic experimental colitis induced by dextran sulphate sodium (DSS) is characterized by Th1 and Th2 cytokines. Clin Exp Immunol. 1998;114(3):385-391.

24. Okayasu I, Hatakeyama S, Yamada M, Ohkusa T, Inagaki Y, Nakaya R. A novel method in the induction of reliable experimental acute and chronic ulcerative colitis in mice. Gastroenterology. 1990;98(3): 694-702.

25. Xue H, Sufit AJ, Wischmeyer PE. Glutamine therapy improves outcome of in vitro and in vivo experimental colitis models. JPEN J Parenter Enteral Nutr. 2011;35(2):188-197.

26. Hu S, Zhu X, Triggs JR, et al. Inflammation-induced, 3'UTRdependent translational inhibition of Hsp70 mRNA impairs intestinal homeostasis. Am J Physiol Gastrointest Liver Physiol. 2009;296(5): G1003-G1011.

27. Hu S, Ciancio MJ, Lahav M, et al. Translational inhibition of colonic epithelial heat shock proteins by IFN-gamma and TNF-alpha in intestinal inflammation. Gastroenterology. 2007;133(6):1893-1904.

28. Liedel JL, Guo Y, Yu Y, et al. Mother's milk-induced Hsp70 expression preserves intestinal epithelial barrier function in an immature rat pup model. Pediatr Res. 2011;69(5 Pt 1):395-400. 


\section{Supplementary figures}

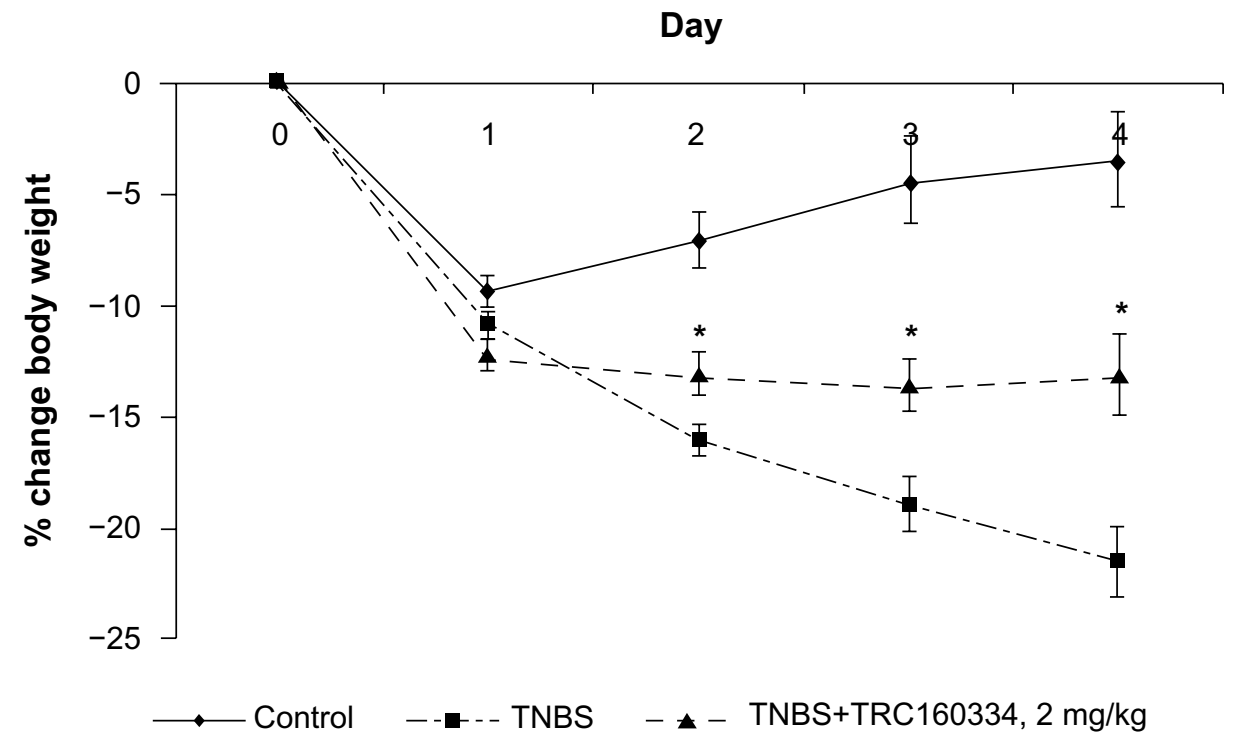

Figure SI Treatment with TRCI 60334 resulted in improvement in the mean percent change body weight profile of male BALB/c mice made colitic using 2,4,6-trinitrobenzene sulfonic acid.

Notes: Data represent mean \pm standard error of the mean. * denotes $P<0.05$ versus vehicle using Student's $t$-test.

Abbreviation: TNBS, 2,4,6-trinitrobenzene sulfonic acid.

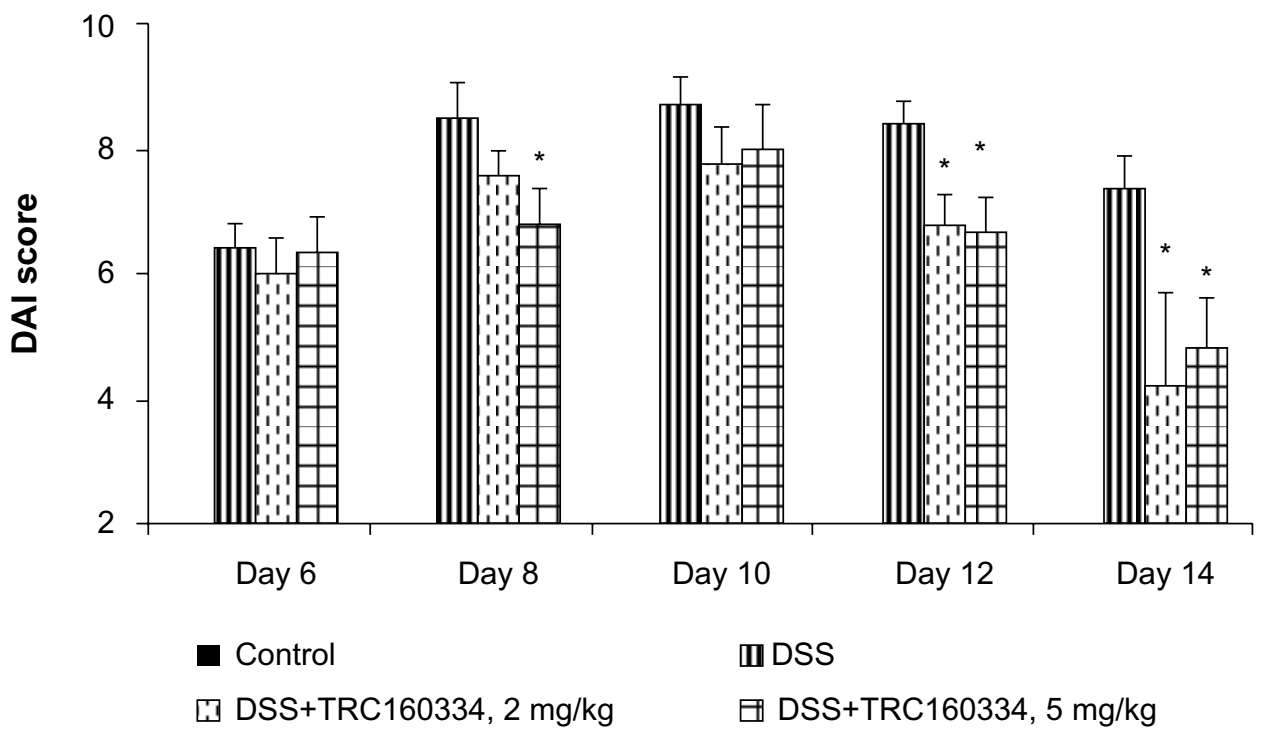

Figure S2 Treatment with TRCI60334 resulted in improvement in mean disease activity index scores of female BALB/c mice made colitic using dextran sulfate sodium. Notes: Data represent mean \pm standard error of the mean. * denotes $P<0.05$ versus the dextran sulfate sodium group using analysis of variance followed by Dunnett's post hoc analysis.

Abbreviations: DSS, dextran sulfate sodium; DAI, disease activity index. 


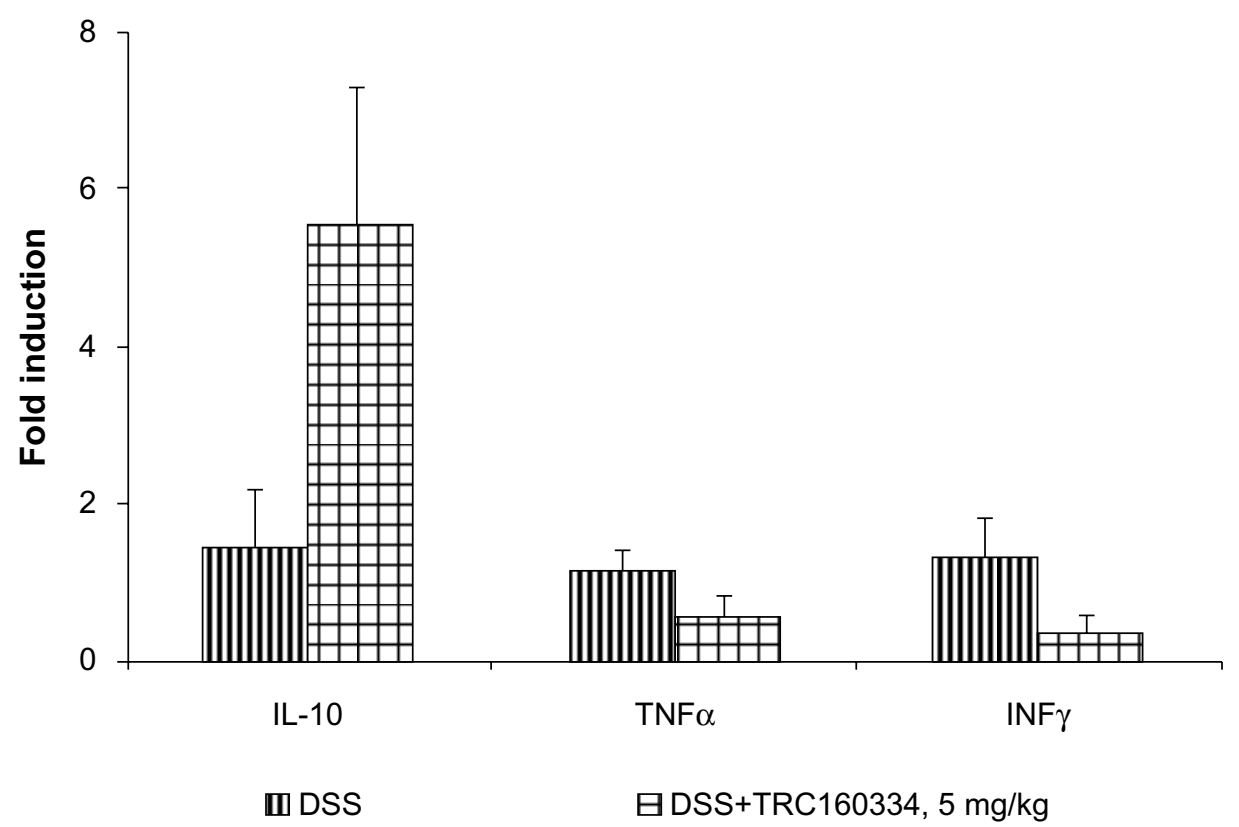

Figure S3 Treatment with TRCI60334 showing trends of reduction in mRNA expression of proinflammatory cytokines tumor necrosis factor $\alpha$ and interferon $\gamma$ and elevation in the expression of the anti-inflammatory cytokine interleukin- 10.

Note: Data represent mean \pm standard error of the mean.

Abbreviations: DSS, dextran sulfate sodium; IL-10, interleukin-10; INF $\gamma$, interferon $\gamma$; TNF $\alpha$, tumor necrosis factor $\alpha$.

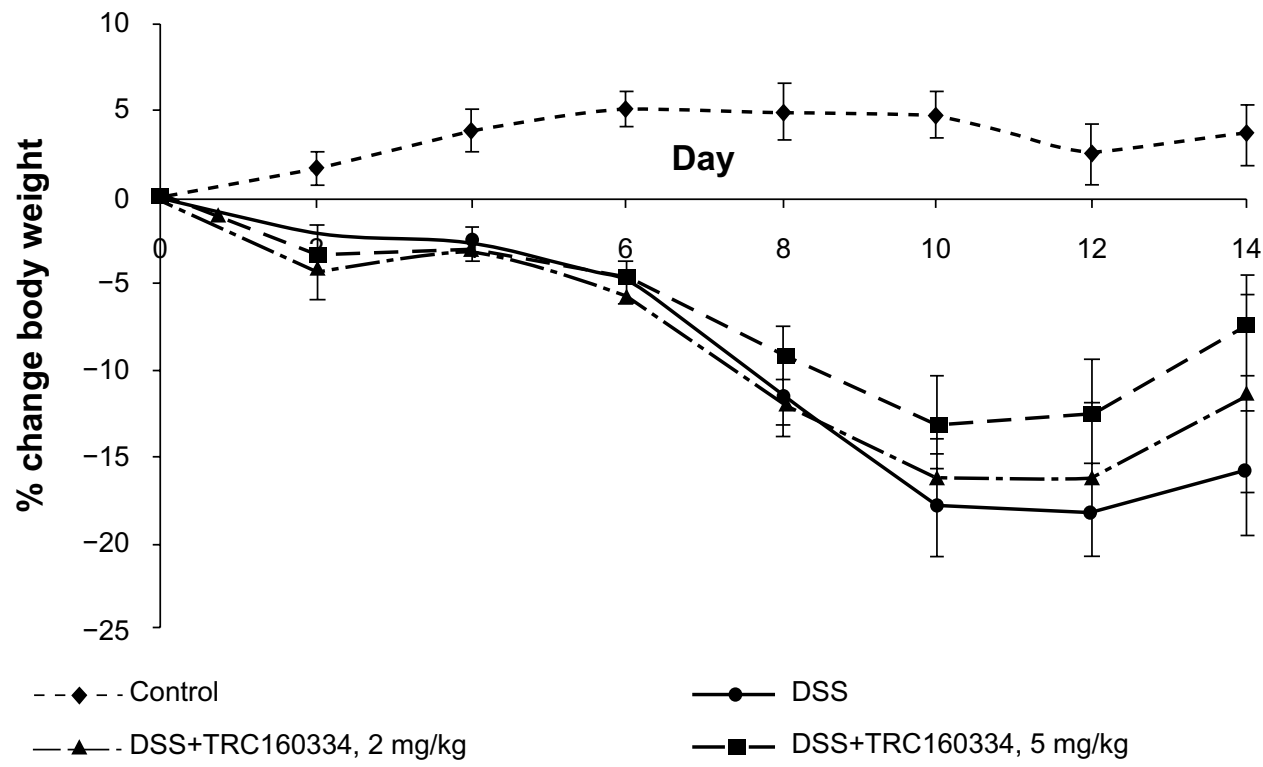

Figure S4 Treatment with TRCI60334 resulted in improvement in the mean percent change body weight profile of female BALB/c mice made colitic using dextran sulfate sodium.

Note: Data represent mean \pm standard error of the mean.

Abbreviation: DSS, dextran sulfate sodium.

Clinical and Experimental Gastroenterology

Dovepress

\section{Publish your work in this journal}

Clinical and Experimental Gastroenterology is an international, peerreviewed, open access journal, publishing all aspects of gastroenterology in the clinic and laboratory, including: Pathology, pathophysiology of gastrointestinal disease; Investigation and treatment of gastointestinal disease; Pharmacology of drugs used in the alimentary tract;
Immunology/genetics/genomics related to gastrointestinal disease. This journal is indexed on CAS. The manuscript management system is completely online and includes a very quick and fair peer-review system. Visit http://www.dovepress.com/testimonials.php to read real quotes from published authors.

Submit your manuscript here: http://www.dovepress.com/clinical-and-experimental-gastroenterology-journal 